\title{
Effets des semis directs de Piliostigma reticulatum sur la régénération de la végétation sur terre dégradée en zone nord soudanienne du Burkina Faso
}

\author{
Yélémou Barthélémy ${ }^{1 *}$; Ouédraogo W. Lucienne ${ }^{2}$; Yaméogo Georges ${ }^{3}$ \\ $1^{*}$ Yélémou Barthélémy, Département Gestion des Ressources Naturelles et Systèmes de Production, INERA \\ CNRST, DRREA-Centre Saria, BP 10 Koudougou, Burkina Faso \\ * Auteur correspondant: Yélémou Barthélémy, E-mail: yelbart@hotmail.com \\ 2. Ouedraogo W. Lucienne, Université Nazi Boni, Dédougou Burkina Faso \\ 3. Yaméogo Georges, Cellule Télédetection et Information Géographique, INERA- CNRST 01 BP 476 Ouagadougou \\ Burkina Faso
}

Original submitted in on $15^{\text {th }}$ May 2018. Published online at www.m.elewa.org on $31^{\text {st }}$ August 2018 https://dx.doi.org/10.4314/jab.v128i1.9

\section{RÉSUMÉ}

Objectif: Piliostigma reticulatum (semellier) est l'une des espèces les plus abondantes dans les jachères soudaniennes du pays. Cette étude vise à situer l'impact des semis directs de $P$. reticulatum sur la régénération des espèces végétales sur terre dégradée.

Méthodologie et résultats: L'étude a été menée dans une parcelle de semis directs de Piliostigma reticulatum ou parcelle principale $\left(400 \mathrm{~m}^{2}\right)$ et trois parcelles témoins de $50 \mathrm{~m}^{2}$ chacune. Dix ans après l'installation de l'essai, l'inventaire de la strate herbacée a été effectué par la méthode des points quadrats. Après la récolte intégrale, la biomasse herbacée a été déterminée. L'effet des semis directs de $P$. reticulatum sur la régénération des espèces ligneuses a été étudié en effectuant un inventaire systématique. La diversité floristique de la parcelle semée est supérieure à celle de la parcelle témoin. De nombreuses espèces sont rencontrées uniquement sous le pied de $P$. reticulatum alors que des espèces telles que Acrocera amplectans Stapf, Cyperus amabilis Vahl, Eragrostis stenela (L.) Roem. \& Schult., Leptadenia hastata (Pers.) Decne, existent seulement dans la parcelle témoin. Le houppier améliore la quantité de la biomasse herbacée de $393 \%$ par rapport au témoin. La régénération ligneuse existe uniquement dans la parcelle principale.

Conclusion et application: Les semis direct de $P$. reticulatum améliorent la diversité floristique, la régénération ligneuse, la production de biomasse. L'utilisation de l'espèce est à promouvoir dans les opérations de végétalisation surtout dans les zones de parcourt du bétail.

Mots clés : Piliostigma reticulatum, semis directs, diversité floristique, biomasse herbacée, Burkina Faso.

\section{ABSTRACT}

Objective: Piliostigma reticulatum (semellier) is one of the most abundant species in Sudanian fallow land. This study aimed to determine the impact of direct seedlings of $P$. reticulatum on woody species regeneration and herbaceous dynamics on degraded lands. 
Methodology and results: The study was conducted in two plots, namely a direct seedling plot of $P$. reticulatum or main plot $(400 \mathrm{~m} 2)$ and three control plots $(50 \mathrm{~m} 2)$. The main plot was planted with $P$. reticulatum in July 2005. Ten years after the installation of the trial, the herbaceous layer survey was conducted using the quadratic point method. After the full harvest, herbaceous biomass was determined. The effect of direct seeding of $P$. reticulatum on the regeneration of woody species was studied by carrying out a systematic inventory. $A$ total of 27 herbaceous species distributed in 11 families and 23 genera were inventoried. Floristic diversity in the main plot was greater than that of the control plot. Some species such as Cassia mimosoides Linn, Desmodium gangeticum (L.) DC., Indigofera suffruticosa Mill, Pennissetum pedicelatum Trin, Tephrosia bracteolata Guill. were encountered only under the crown area of $P$. reticulatum and others such as Acrocera amplectans Stapf, Cyperus, Eragrostis stenela (L.) Roem. \& Schult., Leptadenia hastata (Pers.) Decne in the control plots. Also, the crown improved the herbaceous biomass by $393 \%$ compared to the control. Woody species regeneration occurred only in seeded field.

Conclusion and application of results: Direct seeding of $P$. reticulatum improved floristic diversity, woody species regeneration and biomass production. This species is to be promoted in afforestation and reforestation operations, especially in the areas where cattle are grazed.

Keywords : Piliostigma reticulatum, direct seedling, floristic diversity, herbaceous biomass, Burkina Faso

\section{INTRODUCTION}

Le Burkina Faso, pays à économie rurale, fait face aux défis de l'insécurité alimentaire, de la dégradation des terres et de la pauvreté. L'agriculture, surtout familiale contribue pour $25 \%$ au P.I.B, tandis que l'élevage y est pour $12 \%$, la foresterie et la pêche pour 3\% (MASA, 2013). L'agriculture est donc, la source principale de revenu pour $90 \%$ de la population active du pays (MASA, 2013). Cependant, les fortes pressions démographiques sur les terres agricoles avec des coefficients d'intensité culturale élevés, les techniques agricoles inappropriées (Whitmore, 1997) entrainent une dégradation des ressources naturelles et de l'environnement. Le phénomène de dégradation est telle qu'il faut de plus en plus imaginer des méthodes de lutte peu onéreuses et adaptées à l'échelle des petits producteurs qui sont les plus représentatifs du monde agricole au Burkina Faso. Les semis directs de Piliostigma reticulatum après grattage du sol apparaissent comme une technologie de récupération durable si leurs effets étaient avérés. En effet $P$. reticulatum, de par ses propriétés biologiques et son potentiel socio culturel (Yélémou et al., 2007 ; 2008) est une espèce rustique et adoptée par les populations

\section{MATERIEL ET METHODES}

Site d'étude : La Station de recherche de l'Institut de l'Environnement et de recherches Agricoles (INERA) à rurales. Sa promotion dans un système de récupération des terres dégradées pourrait présenter de nombreux avantages. Cependant les relations de compétition entre les composantes arbre et herbe/cultures ont très souvent été évoquées dans les modèles de fonctionnement et d'aménagement des savanes (Akpo et al., 2009). Pourtant, de nombreux auteurs ont montré que dans les milieux semi-arides, les arbres favorisent non seulement les facteurs microclimatiques, mais augmentent aussi la diversité de la régénération ligneuse, la diversité et la production de la strate herbacée (Apko et al., 2003, 2009 ; Diallo et al., 2015 ; Yélémou et al., 2017). Ainsi, l'effet de l'arbre sur la dynamique herbacée et la régénération ligneuse dépendrait des caractéristiques pluviométriques de la zone d'étude. Cette étude conduite en zone nord soudanienne du Burkina Faso a pour but de définir, d'une part, l'influence des semis direct de $P$. reticulatum sur la structure spécifique de la strate herbacée et la production de phytomasse épigée et, d'autre part, sur l'état de la régénération ligneuse.

Saria a servi de cadre pour cette étude. Elle est située à $80 \mathrm{~km}$ au Nord-Ouest de Ouagadougou la capitale du 
Burkina Faso (Figure 1). Ses coordonnées variations des paramètres atmosphériques, géographiques sont: $12^{\circ} 16^{\prime}$ de latitude nord et $2^{\circ} 09^{\prime}$ pluviométriques, d'humidité et d'évapotranspiration de longitude ouest. Le climat de la zone est de type soudanien marqué, par une forte irrégularité liée aux (Ouédraogo, 2009).

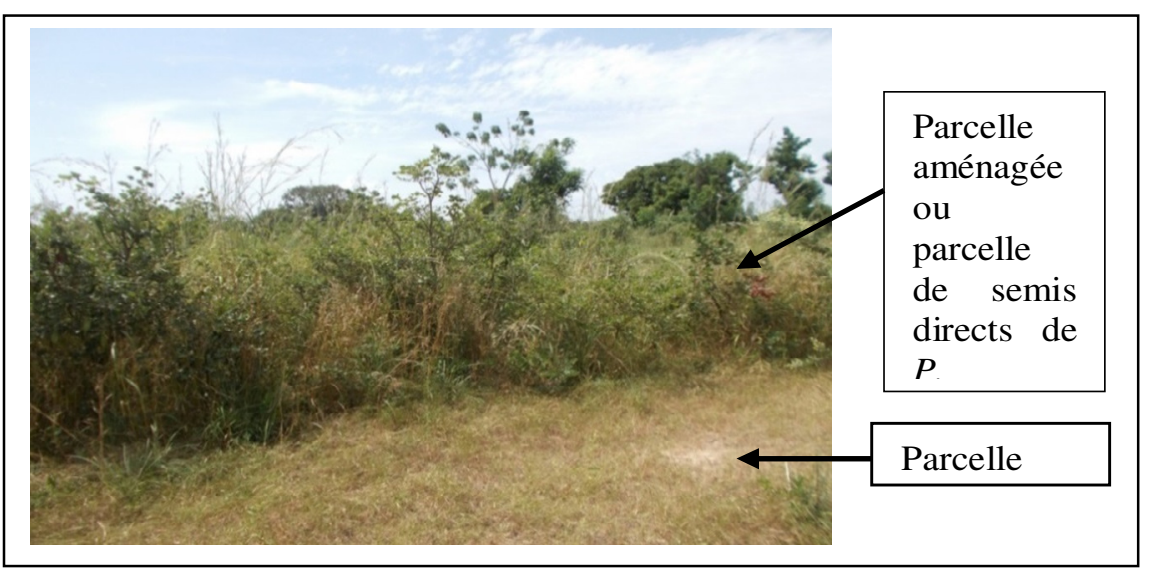

Photo 1 : Parcelle témoin semée densément végétalisé avec un témoin clairsemé d'herbacées de petite taille

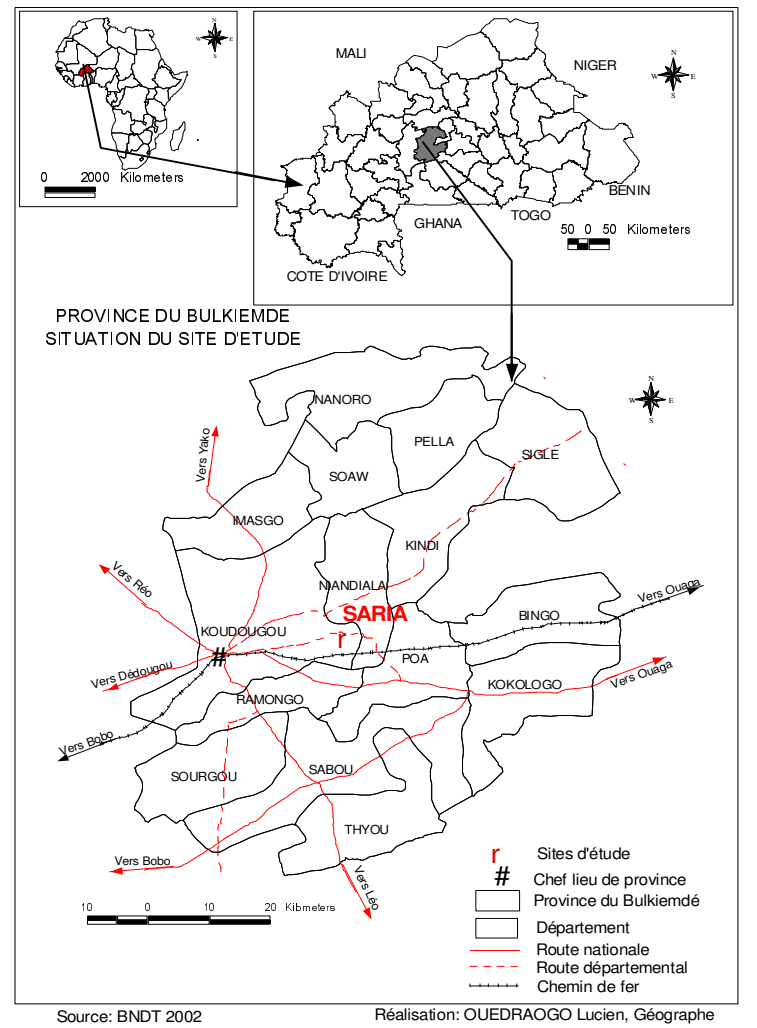

Figure 1: Situation du site de l'étude

Dispositif expérimental : Le dispositif expérimental est constitué de quatre parcelles (Figure 2). Une parcelle principale de $400 \mathrm{~m}^{2}$, ensemencée de $P$. reticulatum en juillet 2005 . Les semis ont été réalisés

en ligne (une graine par poquet). Les poquets sont espacés de $1 \mathrm{~m} \times 1 \mathrm{~m}$. Trois parcelles témoins, de $50 \mathrm{~m}^{2}$ chacune, sont contiguës à la parcelle principale (Photo 1). 


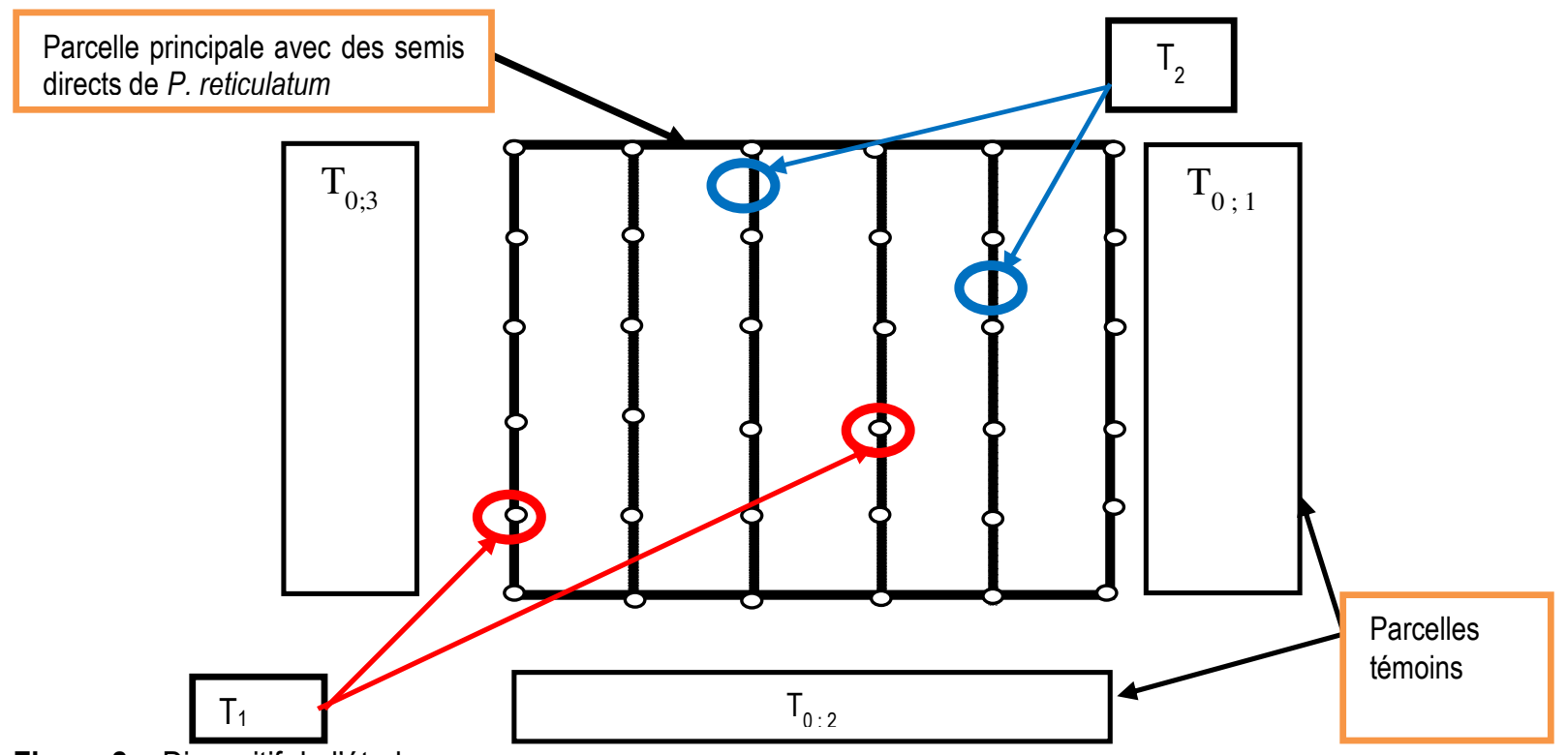

Figure 2 : Dispositif de l'étude

\section{Mesure des paramètres structurels et fonctionnels de la végétation \\ Inventaire floristique de la strate herbacée: La} méthode des points quadrats de Godron et al., (1969) a été utilisée. L'inventaire a été réalisé sur neuf lignes au total soit trois lignes passant sous trois pieds de $P$. reticulatum, trois lignes passant chacune entre deux pieds de $P$. reticulatum, et une ligne par témoin. Ainsi nous avons 300 points de lecture par traitement. Les inventaires ont été effectués en octobre période d'épiaison-fructification des herbacées, et où les espèces sont les plus discernables (Sawadogo, 2009).

Évaluation de la biomasse herbacée : La biomasse herbacée est la masse végétale vivante ou morte sur pied. Elle a été déterminée par la méthode de la récolte intégrale. L'essai a concerné trois (03) pieds de $P$. reticulatum par traitement. Les placettes ont été installés de la manière suivante: $P_{1}=$ placettes sous pied de $P$. reticulatum; $P_{2}=$ placettes entre deux pieds de $P$. reticulatum et $T_{0}=$ placettes témoins. La méthode a consisté à faucher les herbacées dans des placettes de $1 \mathrm{~m}^{2}$ en début du mois d'Octobre. Les herbacées fauchées, ont été triées par espèce. Chaque échantillon d'espèce a été pesé à l'aide d'une balance électronique (Mettler PM 4000g, $d=0,01, e=0,1$ ). Après pesée, les valeurs de la matière sèche ont été déterminées. Ensuite, les échantillons ont été répartis en trois catégories: les graminées annuelles $(\mathrm{Ga})$ et les graminées vivaces(Gv), les légumineuses (Le), et les autres espèces $(\mathrm{Au})$ ou phorbes.
Évaluation de la régénération ligneuse: Les inventaires de la régénération ligneuse ont concerné toute la parcelle aménagée avec $P$. reticulatum et les parcelles témoins. Dans ces placettes toutes les espèces ligneuses ont été notées. Les paramètres dendrométriques ont été enregistrés.

Analyse statistique: Les données floristiques ont permis de calculer des paramètres définis par Daget et Poissonnet (1971) et caractérisant la végétation: la richesse spécifique(S); la fréquence spécifique de l'espèce i $\left(F S_{i}\right)$ qui représente la somme des présences d'une espèce enregistrée sur la ligne; la fréquence centésimale de l'espèce $\mathrm{i}\left(\mathrm{Fc}_{\mathrm{i}}\right)$ avec $\mathrm{F}_{\mathrm{C}_{\mathrm{i}}}=\mathrm{Fsi} / \mathrm{N}^{*} 100$ et $\mathrm{N}$ le nombre de points échantillonnés; la contribution spécifique de l'espèce i $\left(\mathrm{Cs}_{\mathrm{i}}\right)$ avec $\mathrm{Csi}=\mathrm{Fci} / \sum F \mathrm{Fi}$.

Les paramètres de diversité spécifique calculés sont: l'indice de diversité $\left(H^{\prime}\right)$ de Shannon et l'indice d'équitabilité de Piélou $(\mathrm{E})$.

$\mathrm{H}^{\prime}=-\sum p i$. lnpi $\mathrm{Hmax}=\ln \mathrm{N} \quad \mathrm{E}=H^{\prime}$ I Hmax

$H^{\prime}=$ indice de diversité de Shannon, $\mathrm{pi}=$ proportion relative de l'espèce (i),

Hmax =diversité maximale, $\mathrm{N}=$ nombre d'espèces, $\mathrm{In}=$ logarithme népérien. En outre, les données de biomasse herbacée ont été soumises à une analyse de variance à un facteur (ANOVA) en utilisant le test des moindres carrés de Fischer (LSD), afin de comparer les moyennes des différents paramètres sous houppier et hors houppier de $P$. reticulatum au seuil de $5 \%$. Les analyses statistiques ont été réalisées à l'aide du logiciel statistique XIstat version 2007. 


\section{RESULTATS}

Composition floristique: Un total de 27 espèces herbacées représentées dans 11 familles et 23 genres ont été inventoriées dont 17 espèces identifiées dans le témoin et sous le pied de Piliostigma reticulatum. Entre deux pieds de $P$. reticulatum 19 espèces ont été identifiées. On observe une dominance de la famille des Poacées avec 11 espèces soit 40,74\% des espèces inventoriées (Tableau 1).

Tableau 1 : Proportion des espèces par famille

\begin{tabular}{l|c|c}
\hline Famille & Nombre d'espèce & \% des espèces \\
\hline Poacées & 11 & 40,74 \\
Papilionacées & 7 & 25,92 \\
Césalpiniacées & 2 & 7,40 \\
Cypéracées & 1 & 3,70 \\
Acanthacées & 1 & 3,70 \\
Rubiacées & 1 & 3,70 \\
Scrofulariacées & 1 & 3,70 \\
Astéracées & 1 & 3,70 \\
Sterculiacées & 1 & 3,70 \\
Asclépiadacées & 1 & 3,70 \\
\hline
\end{tabular}

On dénombre sept espèces productrices dans les traitements $T_{1}$ et $T_{2}$ contre cinq dans le traitement $T_{0}$ (Tableau 2). Dans le traitement $T_{1}$, les espèces les plus productrices sont Stylosanthes erecta P. Beauv., Andropogon gayanus Kunth., Zornia glochidiata Reighb.ex DC., Andropogon ascinodis C.B. Cl. Quant au traitement $\mathrm{T}_{2}$, Andropogon ascinodis, Andropogon gayanus, Stylosanthes erecta, Zornia glochidiata, et Microchloa indica sont les espèces les plus productrices. Dans le traitement $\mathrm{T}_{0}$, deux espèces, Zornia glochidiata et Microchloa indica ont une productivité élevée.

Tableau 2: Fréquences et contributions spécifiques des espèces productrices selon les traitements

\begin{tabular}{|c|c|c|c|c|c|c|}
\hline Traitement & Nombre d'espèces & Espèces productrices & F.B & Fsi & Fci (\%) & Csi (\%) \\
\hline$T_{0}$ & 17 & $\begin{array}{l}\text { Zornia glochidiata } \\
\text { Microchloa indica } \\
\text { Brachiaria mutica } \\
\text { Stylosanthes erecta } \\
\text { Acrocera amplectans }\end{array}$ & $\begin{array}{l}\text { Le } \\
\text { Ga } \\
\text { Le }\end{array}$ & $\begin{array}{l}284 \\
269 \\
50 \\
55 \\
49\end{array}$ & $\begin{array}{l}94,66 \\
89,66 \\
16,66 \\
18,33 \\
16,33\end{array}$ & $\begin{array}{l}35,19 \\
33,33 \\
6,19 \\
6,81 \\
6,07\end{array}$ \\
\hline $\mathrm{T}_{1}$ & 17 & $\begin{array}{l}\text { Pennisetum pedicelatum } \\
\text { Microchloa indica } \\
\text { Stylosanthes erecta } \\
\text { Brachiaria mutica } \\
\text { Zornia glochidiata } \\
\text { Andropogon ascinodis } \\
\text { Andropogon gayanus }\end{array}$ & $\begin{array}{l}\mathrm{Ga} \\
\mathrm{Ga} \\
\mathrm{Le} \\
\mathrm{Le} \\
\mathrm{Gv} \\
\mathrm{Gv}\end{array}$ & $\begin{array}{l}79 \\
99 \\
130 \\
94 \\
108 \\
104 \\
113\end{array}$ & $\begin{array}{l}26,33 \\
33 \\
43,33 \\
31,33 \\
36 \\
34,66 \\
37,66\end{array}$ & $\begin{array}{l}9,98 \\
12,51 \\
16,43 \\
11,88 \\
13,65 \\
13,14 \\
14,28\end{array}$ \\
\hline $\mathrm{T}_{2}$ & 19 & $\begin{array}{l}\text { Microchloa indica } \\
\text { zornia glochidiata } \\
\text { Brachiaria mutica } \\
\text { Stylosanthes erecta } \\
\text { Andropogon ascinodis } \\
\text { Andropogon gayanus } \\
\text { Pennissetum pedicelatum }\end{array}$ & $\begin{array}{l}\text { Ga } \\
\text { Le } \\
\text { Le } \\
\text { Gv } \\
\text { Gv } \\
\text { Ga }\end{array}$ & $\begin{array}{l}11 \\
112 \\
71 \\
121 \\
159 \\
130 \\
43\end{array}$ & $\begin{array}{l}37 \\
37,33 \\
23,66 \\
40,33 \\
53 \\
43,33 \\
14,66\end{array}$ & $\begin{array}{l}13,3 \\
13,42 \\
8,51 \\
14,5 \\
19,06 \\
15,58 \\
5,27\end{array}$ \\
\hline
\end{tabular}

Légende: FB: Forme Biologique; FSi: Fréquence Spécifique; FCi: fréquence centésimale; CSi: contribution spécifique; Ga : graminée annuelle; Gv : graminée vivace; Le : légumineuse; $T_{0}$ : témoin ; $T_{1}$ : sous pied de Piliostigma reticulatum; $T_{2}$ : entre deux pieds de Piliostigma reticulatum.

Diversité floristique: L'indice de diversité de l'équitabilité de Piélou $(E)$ varient respectivement de Shannon $\left(H^{\prime}\right)$, la diversité maximale $(H \max )$ et 1,77 à 2,22 dans le témoin, de 2,83 à 2,94 sous le pied 
de $P$. reticulatum et de 0,68 à 0,78 entre deux pieds de l'espèce (Tableau 3). On observe que $\mathrm{H}^{\prime}$ ' est supérieur à 1 , que Hmax ne dépasse pas 2,94 et que E est supérieur à 0,5 . Les valeurs de ces indices sont plus importantes sous le pied de $P$. reticulatum qu'entre deux pieds et dans le témoin.

Tableau 3: Diversité floristique par traitement

\begin{tabular}{c|l|l|l|l}
\hline Traitement & Nbre d'espèces & $\mathbf{H}^{\prime}$ (Shannon Wiener) & Hmax & $\mathrm{E}$ (Pielou) \\
\hline $\mathbf{T}_{1}$ & 17 & 2,22 & 2,83 & 0,78 \\
\hline $\mathbf{T}_{2}$ & 19 & 2,20 & 2,94 & 0,74 \\
\hline $\mathbf{T}_{0}$ & 17 & 1,77 & 2,83 & 0,68 \\
\hline
\end{tabular}

Légende : T0 : témoin ; T1 : sous pied de $P$. reticulatum; T2 : entre deux pieds de $P$. reticulatum $H^{\prime}$ : indice de diversité de Shannon, E: indice d'équitabilité de Piélou, Hmax: indice de diversité maximale.

Structure : Sur 17 espèces recensées dans $\mathrm{T} 1,47 \%$ sont des légumineuses et $29 \%$ des graminées annuelles avec des Csi respectives de $36,16 \%$ et $35,65 \%$. La forte Csi chez les légumineuses est due surtout à Stylosanthes erecta (Csi $=16,43 \%)$ et ensuite à Zornia glochidiata (Csi $=13,65 \%)$. Chez les graminées annuelles, le pourcentage élevé de Csi est due à Microchloa indica avec $12,51 \%$ de Csi et à Brachiaria mutica avec $11,88 \%$ de Csi. Dans le traitement $\mathrm{T}_{2}, 42 \%$ sont des graminées annuelles et $37 \%$ des légumineuses sur les 19 espèces inventoriées. Dans le témoin, bien que les légumineuses et les graminées annuelles sont

Tableau 4: Proportion des différentes strates herbacées (\%) suivant les traitements

\begin{tabular}{c|c|c|c}
\hline Strates & T1 & T2 & T0 \\
\hline $\mathbf{0 - 2 0} \mathbf{c m}$ & 7,4 & 16,66 & 40 \\
\hline $\mathbf{2 0}-\mathbf{4 0} \mathrm{cm}$ & 25,92 & 41,66 & 55 \\
$\mathbf{4 0 - 6 0 \mathrm { cm }}$ & 29,62 & 8,33 & 5 \\
\hline $\mathbf{6 0 - 8 0} \mathbf{c m}$ & 3,7 & 0 & 0 \\
\hline $\mathbf{8 0 - 1 0 0} \mathbf{c m}$ & 0 & 0 & 0 \\
\hline$>100 \mathrm{~cm}$ & 33,33 & 33,33 & \\
\hline
\end{tabular}

Légende : T0 : témoin ; T1 : sous pied de $P$. reticulatum; T2 : entre deux pieds de $P$. reticulatum

Effet de $P$. reticulatum sur la production de la biomasse herbacée : L'analyse de la variance a donné des différences très significatives $(p<0,002)$ entre les traitements (Tableau 5). En effet, dans le traitement $\mathrm{T}_{1}$, représentées chacune par 6 espèces, leur Csi est de $52,42 \%$ pour les légumineuses et de $41,88 \%$ pour les graminées annuelles.

Stratification du couvert végétal : Dans le témoin $\left(T_{0}\right)$, il n'y a pas d'individus ayant une hauteur supérieure à $60 \mathrm{~cm}$ (Tableau 4). Aussi on observe des proportions élevées des individus dont les hauteurs sont comprises entre 0 à $40 \mathrm{~cm}$. Dans les deux autres traitements $\left(T_{1}\right.$ et $\left.T_{2}\right)$, on observe aussi bien des individus de faible hauteur que des individus ayant une hauteur $>100 \mathrm{~cm}$.

Tableau 5 : Production épigée $\left(\mathrm{g} / \mathrm{m}^{2}\right)$ selon les traitements

\section{Biomasse épigée moyenne (g pour $\left.1 \mathrm{~m}^{2}\right)$}

\begin{tabular}{l|l}
\hline Traitement & Quantité $(\mathrm{g})$ \\
$\mathrm{T}_{1}$ & $359,33 \pm 83,72^{\mathrm{a}}$ \\
$\mathrm{T}_{2}$ & $238,33 \pm 15,94^{\mathrm{b}}$ \\
$\mathrm{T}_{0}$ & $91,33 \pm 15,30^{\mathrm{c}}$ \\
$\mathrm{Pr}>\mathrm{F}$ & 0,002 \\
Significativité & $\mathrm{S}$ \\
\hline
\end{tabular}

Légende : $T_{0}$ : témoin $; T_{1}$ : sous pied de Piliostigma reticulatum ; $T_{2}$ : entre deux pieds de Piliostigma reticulatum. Dans la même colonne, les nombres suivis de la même lettre ne sont pas significativement différents au seuil de $5 \%$ selon le test de Fischer 
État de la régénération ligneuse : La régénération ligneuse est seulement notée dans la parcelle de semis directs de $P$. reticulatum. Des espèces inventoriées, Khaya senegalenis (Ders.) A. Juss. et Guiera senegalensis J.F. Gmel sont les plus présentes. La distribution de la régénération ligneuse par classe de hauteur [0-1m [, [1-2m [ et supérieure ou égale à $2 \mathrm{~m}$,

\section{DISCUSSION}

Composition floristique: La richesse floristique est plus élevée dans le traitement $T_{2}$, que dans les autres traitements $\left(T_{0}\right.$ et $\left.T_{1}\right)$. Le nombre élevé d'espèces dans le traitement $T_{2}$ pourrait être dû à l'amélioration des conditions favorables au développement des espèces herbacées. Yélémou et al., (2017), avaient trouvé que la richesse floristique herbacée sous couvert de $P$ reticulatum est significativement plus élevée que celle hors couvert, au niveau des formations à $P$. reticulatum de plus de 15 ans avec un espacement de $5 \mathrm{~m} \times 5 \mathrm{~m}$. La faible richesse spécifique dans le traitement $T_{1}$ par rapport au traitement $T_{2}$, pourrait s'expliquer par l'influence directe du houppier (Traoré et al., 2007 ; Yélémou et al., 2007). Aussi la proximité des pieds de $P$. reticulatum $(1 \mathrm{~m} / 1 \mathrm{~m})$ et la petite taille des individus de l'espèce pourraient expliquer la faible expression de la richesse floristique dans le traitement $\mathrm{T}_{1}$. Cependant, dans le traitement $T_{1}$, nous notons la présence des espèces telles que Andropogon gayanus, Andropogon ascinodis, Pennissetum pedicellata qui montrent une amélioration de la fertilité du sol (Somé et al., 2006). En effet le houppier permet de réduire l'évapotranspiration potentielle mais aussi augmente la fertilité du sol (Traoré et al., 2007). Quant au traitement témoin ( $\left.T_{0}\right)$, la faible richesse spécifique s'explique par la forte dégradation du sol qui empêche le développement de nombreuses espèces. La présence élevée des espèces telles que Zornia glochidiata et Microchloa indica, témoigne de l'état de cette dégradation. Ainsi, plus on se rapproche du houppier de l'espèce, plus la diversité spécifique augmente. Cette forte diversité sous $P$. reticulatum et entre deux pieds de $P$. reticulatum serait due aux teneurs élevées en matières organiques (Tyano et al., 2016).

Structure: Les graminées annuelles représentent le groupe le plus important. La prédominance des graminées annuelles dans notre site concorde avec beaucoup d'auteurs (Sawadogo, 1996 ; Doulcom, 2000 ; Mbayngone, 2008 ; Ouédraogo, 2009) qui ont montré la dominance de ces dernières dans la zone soudanienne. Leur prédominance est due à la facilité qu'elles ont de se développer dans les conditions laisse voir que, 10 ans après semis de $P$. reticulatum, $57,14 \%$ de la régénération est inférieure à $1 \mathrm{~m}$ de hauteur et $28,57 \%$ se répartit entre 1 et $2 \mathrm{~m}$. En outre, les individus de hauteur égale ou supérieure à $2 \mathrm{~m}$ ne représentent que $14,30 \%$ de la régénération et ce sont des individus de l'espèce Guiera senegalensis.

difficiles mais aussi parce qu'elles sont moins exigeantes. C'est dans ce sens que Ouédraogo (2008) disait que : " les graminées annuelles sont des espèces moins exigeantes pouvant se développer sur des sols peu fertiles ». Les graminées vivaces sont faiblement représentées dans la zone d'étude mais aussi dans les différents traitements. Ces résultats corroborent ceux de Yanra (2004) et Ouédraogo (2008) qui ont montré que dans la zone soudanienne la part des graminées vivaces dans le couvert herbacé est faible. Cette faible représentativité s'expliquerait par la faible aptitude qu'elles ont de résister aux mauvaises conditions climatiques et aux pressions anthropiques. L'apparition des espèces telles que Andropogon ascinodis, Andropogon gayanus, Pennissetum pedicelatum montre une amélioration du niveau de fertilité du sol dans la parcelle semée, puisque ces espèces ont des exigences nutritionnelles élevées. En outre, la faible Csi de Microchloa indica sous $P$. reticulatum et entre deux pieds $P$. reticulatum par rapport au témoin et de Zornia glochidiata sous $P$. reticulatum et entre deux pieds de $P$. reticulatum comparé au traitement $T_{0}$ témoigne de l'augmentation de la fertilité au niveau de la parcelle semée. En effet, Microchloa indica et Zornia glochidiata sont des espèces indiquant la dénudation du sol donc sont caractéristiques des sols dégradés. Ainsi $P$. reticulatum contribue à la lutte contre la dénudation du sol en réduisant la Csi de Microchloa indica et de Zornia glochidiata.

Effet de $P$. reticulatum sur la production de la biomasse herbacée : Les semis de $P$. reticulatum ont permis une augmentation significative $(p<0,05)$ de la production de la phytomasse herbacée. En effet, sous $P$. reticulatum et entre deux pieds de $P$. reticulatum la quantité de biomasse produite est élevée par rapport au témoin. Ces résultats sont similaires à ceux obtenus par Yélémou et al., (2017) avec la même espèce. Andropogon gayanus, Andropogon ascinodis sont à l'origine de cette grande production de phytomasse dans la parcelle aménagée. L'apparition de ces espèces est la preuve de l'amélioration de la fertilité du 
sol (Somé et al., 2006 ; Bilgo et al., 2006). Également l'importante production de biomasse serait due à la diversité floristique qui est plus élevée sous $P$. reticulatum que dans le témoin.

Effet de $P$. reticulatum sur la régénération ligneuse : Nos résultats indiquent que $P$. reticulatum contribue à la régénération des espèces ligneuses. La régénération ligneuse est plus exigeante en eau et en éléments fertilisants du sol que la régénération herbacée. Or le témoin par la présence des espèces telles que

\section{CONCLUSION}

Les semis directs de $P$. reticulatum restaurent la dégradation des terres et la fertilité des sols. En effet, l'indice de diversité de Shannon indique une forte diversité herbacée dans la parcelle aménagée. $P$. reticulatum contribue à la lutte contre la dénudation du sol en réduisant la Csi de Microchloa indica et de Zornia glochidiata. En outre, les semis de $P$. reticulatum

\section{REFERENCES BIBLIOGRAPHIQUES}

Akpo LE et Grouzis M: 2009. Effet des arbres sur la diversité de la végétation

herbacée dans les parcours communautaires du Nord Sénégal (Afrique de l'Ouest), pp. 272-293.

Akpo LE, Banoin M et Grouzis M: 2003. Effet de l'arbre sur la production et la qualité fourragères de la végétation herbacée : bilan pastoral en milieu sahélien. Revue Méd Vét., 154: 619-628.

Bilgo A, Masse D, Sall S, Serpantie G, Chotte JL et Hien V: 2006. Chemical and microbial properties of semiarid tropical soils of shortterm fallows in Burkina Faso, West Africa. Biology and fertility of soils, 43: 313-320.

Botoni/Liehoun E, Daget $P$ et César J: 2006. Activités de pâturage, biodiversité et végétation pastorale dans la zone Ouest du Burkina Faso. Revue Élev. Méd. vét. Pays trop., 59: 31-38.

Daget $P$ et Poissonet J: 1971. Un procédé d'estimation de la valeur pastorale des pâturages. Fourrages: 31-39

Diallo M, Ndiaye O, Diallo A, Saleh M, Bassene C, Wood SA, Diop A \& Guisse A: 2015. Influence de la litière foliaire de cinq espèces végétales tropicales sur la diversité floristique des herbacées dans la zone du Ferlo (Sénégal). Int. J. Biol. Chem. Sci. 9: 803-814.

Doulcom G: 2000. Problématique des espaces agrosylvopastoraux dans la province du Bam :
Microchloa indica et de Zornia glochidiata montre toujours un état de dégradation du sol impropre à la régénération ligneuse. Par contre $P$. reticulatum, aussi bien par son houppier que par son système racinaire contribue à l'amélioration de la fertilité des sols (Yélémou et al., 2007 ; 2008). Grouzis et Le Floc'h, (2003) ont montré aussi d'importante régénération sous Acacia raddiana, Balanites aegyptiaca et Ziziphus mauritiana.

créent une importante phytomasse herbacée favorable à l'installation de la régénération ligneuse. Au regard de ces résultats, l'espèce est à conseiller dans les processus de plantation d'arbre visant à récupérer les terres dégradées, à améliorer la biodiversité et la production herbacée.

le cas de la relique de brousse de Tanlili. Mémoire d'ingenieur, IDR/UPB, 113p.

Godron M, Daget P, Emberger L, Long G, Le floc'h E, Poissonet J, Sauvage Ch \& Wacquant JM: 1969. Vade-mecum pour le relevé méthodique de la végétation et du milieu. C.N.R.S. Paris, $170 \mathrm{p}$.

Grouzis M et Le Flochk: 2003.Un arbre au désert: Acacia raddiana. IRD, Paris. $313 p$

MASA: 2013. Quelle stratégie pour le renforcement de la résilience des populations face au changement climatique en vue d'assurer une sécurité alimentaire durable? Journée Nationale du Paysan (I6ème édition, 2013), Burkina Faso. $16 \mathrm{p}$.

Mbayngone $E: 2008$. Flore et végétation de la réserve partielle de faune de Pama, sud-est du Burkina Faso. Thèse de doctorat, Univ. Ouaga. $137 \mathrm{p}$.

Ouédraogo 0: 2009. Phytosociologie, dynamique et productivité de la végétation du Parc national d'Arly (Sud-est du Burkina Faso).Thèse de doctorat, Univ. Ouaga.140p.

Ouédraogo D: 2008. Caractérisation des ressources fourragères et des pratiques pastorales $\mathrm{du}$ terroir de Kotchari à la périphérie du parc $w$. Mémoire d'ingénieur UPB/IDR. $87 \mathrm{p}$.

Salawu A: 2009. Influence des modes de gestion de la fertilité des sols sur l'activité microbienne dans un système de cultures de longue durée au 
Burkina Faso. Doctorat d'état ès-sciences naturelles. Université Polytechnique de Bobo, Burkina Faso. 201 p.

Sawadogo L: 2009. Influence des facteurs anthropiques sur la dynamique de la végétation des forêts classées de Laba et de Tiogo en zone soudanienne du Burkina Faso. Thèse Doctorat d'État, Université de Ouagadougou. $142 p$

Some NA, Hien V \& Ouédraogo SJ: 2006. Amélioration du statut organique d'un sol ferrugineux tropical lessivé sous jachère artificielle à Andropogon spp. au Burkina Faso : effet des traitements sur la chimie du sol. Tropicultura 24: 200-207.

Traoré S, Thiombiano L, Millogo JR \& Guinko S : 2007. Carbon and nitrogen enhancement in Cambisols and Vertisols by Acacia spp. in eastern Burkina Faso: Relation to soil respiration and microbial biomass. Applied Soil Ecology 35: 660-669.

Tyano A, Yélémou B and Bationo BA: 2016. Impacts of $P$. reticulatum on chemical and biological properties of fallows in sudanian zone of Burkina faso. International Journal of Current Research 8: 41878-41885

Whitmore TC: 1997. Tropical forest disturbance, disappearance, and species loss. In: Laurence
W. F. et Bierregaard R. O. (eds) Tropical Forest Remmants Ecology, Management and Conservation of Fragmented Communities. Chicago Illinois: 3-12.

Yanra JO: 2004. Caractérisation des pâturages naturels en zone sud soudanienne du Burkina Faso: Cas des terroirs de Sidi, Guena et Banfoulagué dans la province du Kénédougou. Mémoire de fin d'étude. IDR/UPB, Burkina Faso. 77p.

Yélemou B, Bationo BA, Yaméogo G. \& MillogoRasolodimby J: 2007. Gestion traditionnelle et usage de Piliostigma reticulatum (D.C.) Hochst., dans le plateau central du Burkina Faso. Bois et For. Trop. 291: 55-65.

Yélémou B, Tyano A, Bationo BA, Ouattara B, Koala J. \& Millogo Rasolodimby J : 2017. Effects of Piliostigma reticulatum on the vegetation dynamic in sudanian zone of Burkina Faso, Journal of Plant Studies. 6: 77-93.

Yélémou $B$, Yaméogo $G$, Bationo $B A$, Millogo/Rasolodimby $\mathrm{J}$ et Hien V: 2008. Biologie florale et mode de reproduction sexuée de Piliostigma reticulatum, (DC) Hochst. Int. J. Biol. Chem. Sci. 2 : 281-291. 